\title{
Elastic scattering of electrons by barium atoms using the distorted wave method
}

\author{
Kago J. N. ${ }^{1}$, Okumu J. ${ }^{1}$, Singh C. S. ${ }^{{ }^{*}}$ \\ ${ }^{1,1^{*}}$ Department of Physics, Kenyatta University, P.O Box 43844-0100, Nairobi, Kenya \\ ${ }^{1 *}$ Corresponding Author. Email: singh.chadra@ku.ac.ke
}

Received: 10 July 2020 / Accepted: 26 September 2020 / Published online: 20 November 2020

\begin{abstract}
Differential and integral cross sections of elastic scattering of electrons by barium atoms at intermediate energies $(10-200 \mathrm{eV})$ were calculated using the distorted wave method. Being an elastic scattering both the initial and final distortion potentials were taken as the static potential of a barium atom in the initial state. The distorted waves are determined by the partial wave expansion method by expanding them in terms of spherical harmonics while the radial equation corresponding to distorted waves is evaluated using the Numerov method. A computer program DWBA1, for $\mathrm{e}^{-}-\mathrm{H}$ scattering was modified to perform numerical calculations for $e^{-}-B a$ scattering and the results obtained are compared with the experimental and theoretical results available. The present integral cross sections are in good qualitative agreement with the experimental results and most of the theoretical results. For energies in the range $30-100 \mathrm{eV}$ the present differential cross sections are in satisfactory agreement with the other theoretical and experimental results.
\end{abstract}

Key words: differential cross section; integral cross section; barium atom; distorted wave method.

\section{INTRODUCTION}

Theory of atomic collisions is crucial for theoretical as well as experimental studies in atomic physics, in particular cross sections for electron-barium scattering are needed for modeling the behavior of Ba lasers, discharge lamps, plasma switches and ionosphere where $\mathrm{Ba}$ has often been used as a trace element for diagnostic purposes. On the academic side, benchmark laboratory cross sections are needed for testing various theoretical approximations and calculation methods attempting to predict these cross sections.

Over the last three decades there have been both theoretical and experimental works on the calculation of cross sections for elastic scattering of electron by barium atom. Jensen et al. (1978) measured elastic scattering cross sections for energies between 20 and $100 \mathrm{eV}$. Wang et al. (1994) also measured elastic cross sections for energies between 5 and $20 \mathrm{eV}$. Trajmar et al. (1999) measured the elastic cross sections at $30 \mathrm{eV}$. The theoretical calculations include those by Fabrikant (1980) for a few energies using the two state close coupling method (CC2), by Szmytkowski and Sienkiewicz (1994) for energies between 15 and $100 \mathrm{eV}$ using the relativistic polarized orbital method, the convergent close coupling (CCC-115) calculations by Fursa and Bray (1999), the phase theory by Miloshevsky et al. (2000) for energies between 15 and $100 \mathrm{eV}$, by Adibzadeh and Theodosiou (2004) using the potential scattering method, by Kelemen et al. (1995) for energies between 0.1 to $200 \mathrm{eV}$ and the semi-relativistic approach used by Kumar et al. (1994). However, there is inconsistency between experimental and theoretical results and between theoretical results themselves. This shows there is a gap and further work is needed.

In this paper we present the calculation of differential and integral cross sections of elastic scattering of electrons by barium atoms using the distorted wave method (DWM). A primary advantage of this method lies in the fact that more of the important physical effects may be included in the leading terms of the perturbation series expansion such that the distorted wave series converges faster than the Born series. Consequently, reasonably accurate results may be obtained from the first term of the distorted wave series. 
In order to evaluate the direct and exchange transition matrices we used the Roothan-Hatree-Fock (RHF) double zeta functions given in the McLean and McLean (1981) tables. The double zeta function is an approximate RHF function in which a given electron orbital is described by two Slater functions. For numerical calculations we have used a computer program DWBA1 by Madison and Bartschat (1996) that was written for electron-hydrogen scattering. The code was modified to fit electron-barium scattering. The present results are compared with the available experimental and theoretical results.

\section{THEORY}

The total Hamiltonian, $\mathrm{H}$, for electron-barium scattering can be written as

$H=H_{a}+T+V$

where $H_{a}$ is the Hamiltonian of the isolated atom, T the Hamiltonian for the projectile electron (kinetic energy operator) and $\mathrm{V}$ is the interaction between the projectile and atom, given as (in atomic units)

$V=\frac{-N}{r_{0}}+\sum_{i=1}^{N} \frac{1}{r_{0 i}}$

where $N$ is the atomic number of the atom, $r_{0}$ is the distance of the projectile electron from the nucleus of the target and $r_{0 i}$ is the distance of the projectile from the $i^{t h}$ atomic electron.

The initial state full scattering wave function $\Psi_{i}^{+}$ representing the projectile-atom system is a solution of the Schrödinger equation

$(H-E) \Psi_{i}^{+}=0$

where the + superscript indicates the outgoing wave boundary conditions (asymptotically it is given as the initial atomic wavefunction times the asymptotic form the incident plane wave + outgoing scattered spherical wave).

The T-matrix for the electron-target (N-electron atom) collision problem in the two-potential approach $(V$ is decomposed as $(V-U)+U$ ) can be written as (Madison \& Bartschat, 1996)

$T_{i f}=(N+1)\left\langle\chi_{f}^{-}(0) \psi_{f}(1, ., N)\left|V-U_{f}\right| A \Psi_{i}^{+}(0, . ., N)\right\rangle$

$+\left\langle\chi_{f}^{-}(0) \psi_{f}(1, \ldots N)\left|U_{f}\right| \psi_{i}(1, \ldots, N) \beta_{i}(0)\right\rangle$

where $\beta_{i}$ is the initial state plane wave of the projectile electron, given as,

$\beta_{i}=\exp \left(i k \cdot r_{0}\right)$

where $\psi_{i}$ and $\psi_{f}$ are respectively, initial and final wave functions of the barium atom. $A$ is the antisymmetrization operator for the electron-atom system which can be expressed as

$A=\frac{1}{N+1}\left(1-\sum_{i=1}^{N} P_{i 0}\right)$

where $P_{i 0}$ is the operator that exchanges the $i^{\text {th }}$ atomic electron with the incident electron (denoted by 0 ).

$\chi_{f}^{-}$is the distorted wave satisfying the incoming wave boundary condition and is a solution of the wave equation

$$
\left(\nabla_{0}^{2}+2 U_{f}-2 k_{f}^{2}\right) \chi_{f}^{-}=0
$$

where $k_{f}$ is the final state wave vector of the projectile $\left(k_{f}^{2}\right.$ gives the kinetic energy (in atomic units) of the projectile in its final state).

$U_{f}$ is an arbitrary potential (which is generally chosen as a static potential $\langle\psi|V| \psi\rangle$ of the target) for distortion for the final projectile electron wavefunction.

$\Psi_{i}^{+}$in equation (4) cannot be evaluated exactly and hence the need for more approximation. When it is expressed as the product of initial state distorted waves $\chi_{i}^{+}$times an initial atomic wave function $\psi_{i}$, it gives the first order distorted wave Born approximation to the T-matrix given by equation (4). $\Psi_{i}^{+}$is written as

$\Psi_{i}^{+}(0,1, \ldots, N)=\psi_{i}(1,2, \ldots N) \chi_{i}^{+}(0)$

where $\chi_{i}^{+}$is the distorted wave function representing the projectile electron in the initial state and is a solution to the wave equation

$\left(\nabla_{0}^{2}+2 U_{i}-2 k_{i}^{2}\right) \chi_{i}^{+}=0$.

Using equations (6) and (8) in equation (4) and considering a case of elastic scattering of an electron by a two electron atom (in the outermost shell of a barium atom there are two electrons and assuming that the inner core is frozen), we get the transition matrix element $T_{i f}$ in terms of the direct $\left(T_{i f}^{a}\right)$ and exchange $\left(T_{i f}^{\theta x}\right)$ scattering amplitudes as

$T_{i f}=T_{i f}^{d}-T_{i f}^{e x}$

where

$T_{i f}^{d}=\left\langle\chi_{f}^{-}(0) \psi_{i}(1,2)\left|U_{f}\right| \psi_{i}(1,2) \beta_{i}(0)\right\rangle$

and

$T_{i f}^{e x}=-\left\langle\chi_{f}^{-}(0) \psi_{f}(1,2)\left|V-U_{f}\right| \psi_{i}(0,2) \chi_{i}^{+}(1)+\psi_{i}(1,0) \chi_{i}^{+}(2)\right\rangle$.

Since $\psi_{i}=\psi_{f}$ the first term in equation (4) vanishes for direct scattering, assuming the orthogonality of distorted waves. When making calculations we are going to neglect the term associated with $U_{f}$ in $T_{i f}^{e x}$, assuming that the 
overlap integral between the bound state and distorted wave will be negligible because of the oscillatory nature of the distorted wave. The $T_{i f}^{\theta x}$ then takes the form

$T_{i f}^{\theta x}=-\left\langle\chi_{f}^{-}(0) \psi_{f}(1,2)|V| \psi_{i}(0,2) \chi_{i}^{+}(1)+\psi_{i}(1,0) \chi_{i}^{+}(2)\right\rangle$.

To evaluate the direct and exchange scattering amplitudes, the distorted waves $\chi_{i}^{+}$and $\chi_{f}^{-}$are expanded in term of the partial waves as

$\left|\chi_{i}^{+}\right\rangle=\sqrt{\frac{2}{\pi}} \frac{1}{k_{i} r} \sum_{l_{i} m_{i}} i_{i}^{l_{i}} \chi_{l_{i}}\left(k_{i}, r\right) Y_{l_{i} m_{i}}(\hat{r}) Y_{l_{i} m_{i}}^{*}\left(\widehat{k}_{i}\right)$

and

$\left|\chi_{f}^{-}\right\rangle=\sqrt{\frac{2}{\pi}} \frac{1}{k_{f} r} \sum_{l_{f} m_{f}} i^{l_{f}} \chi_{f_{f}^{*}}^{*}\left(k_{f}, r\right) Y_{l_{f} m_{f}}(\hat{r}) Y_{l_{f} m_{f}}^{*}\left(\widehat{k}_{f}\right)$

where $Y_{l m}$ are spherical harmonics and $\hat{r}$ and $\widehat{k}$ are unit vectors denoting the direction of $\mathbf{r}$ and $\mathbf{k}$. In the expansion of $\chi_{f}^{-}$the complex conjugate of the radial part $\chi_{l_{f}}$ is taken so that it satisfies the incoming wave boundary condition. Substituting the above partial wave expansions of the distorted waves in equations (7) and (9) we find that the radial distorted waves are solutions of the following equation

$\left(\frac{d^{2}}{d r^{2}}-\frac{l_{s}\left(l_{s}+1\right)}{r^{2}}-U_{s}(r)+k_{s}^{2}\right) \chi_{l}(r)=0$

with $s=\mathrm{i}$ for the initial state and $s=\mathrm{f}$ for the final state distorted waves. In the asymptotic region, they satisfy the boundary condition

$\lim _{r \rightarrow \infty} \chi_{l_{s}}\left(k_{s}, r\right)=j_{l_{s}}+B_{l_{s}}\left(-\eta_{l_{s}}+i j_{l_{s}}\right)$

where $j_{l}$ and $\eta_{l}$ are regular and irregular Ricatti-Bassel functions and $B_{l}$ is given as

$B_{l}=\exp \left(i \delta_{l}\right) \sin \delta_{l}$

where $\delta_{l}$ is the elastic scattering phase shift.

The radial distorted wave equation (16) for initial and final states are solved by using the Numerov method (Madison and Bartschat, 1996) and the differential cross section (DCS) was obtained using the relation

$\frac{d \sigma}{d \Omega}=4 \pi^{4} \frac{k_{f}}{k_{i}}\left(\left|T^{d}-T^{e x}\right|^{2}\right)$.

The integral cross section $\sigma$ was obtained by using the formula

$\sigma=\int \frac{d \sigma}{d \Omega} d \Omega=2 \pi \int_{0}^{\pi} \frac{d \sigma}{d \Omega} \sin \theta d \theta$.

We have used the modified form of the computer program DWBA1 written by Madison and Bartschat (1996) to evaluate the direct and exchange matrix elements and then the differential and integral cross sections. The original program is written for electron-hydrogen scattering; we modified it for electron-barium scattering.

\section{RESULTS AND DISCUSSION}

The model presented above was used to calculate the differential and integral cross sections for elastic scattering of electrons by barium atoms for impact energies of 10 , $20,30,40,60,80,100$ and $200 \mathrm{eV}$. To test the reliability of our model we compared the results with theoretical and experimental data available. Results of such comparison for differential cross section for elastic scattering of electron from barium atom are shown in Figures $1-9$ and results for integral cross section are shown in Figure 10.

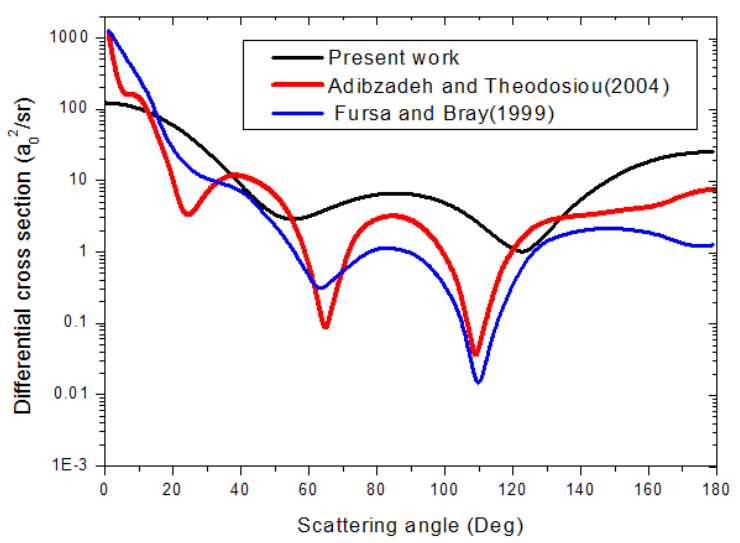

Figure 1. Differential cross section for elastic scattering of electron by barium atom at $10 \mathrm{eV}$ incident energy.

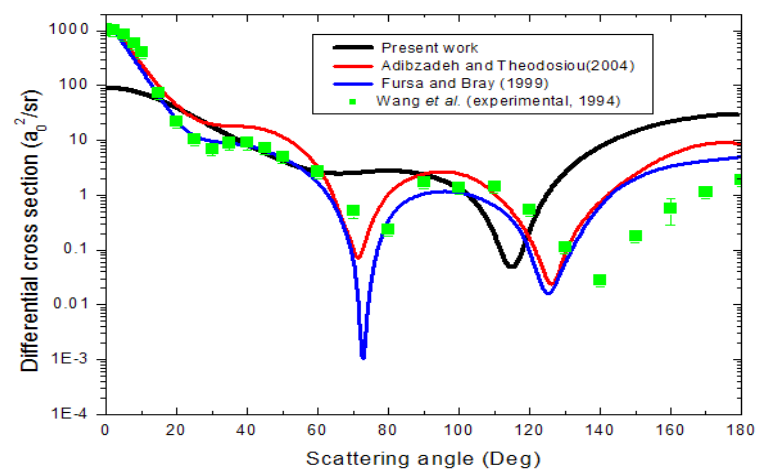

Figure 2. Differential cross section for elastic scattering of electron by barium atom at $15 \mathrm{eV}$ incident energy.

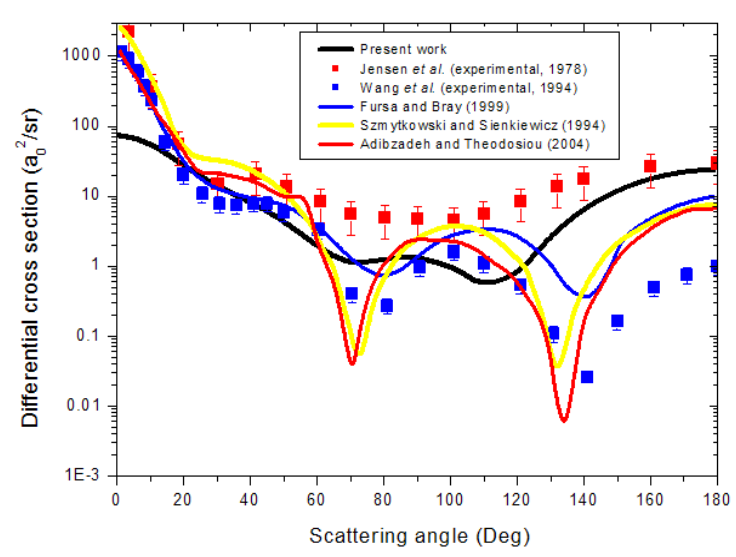

Figure 3. Differential cross section for elastic scattering of electron by barium atom at $20 \mathrm{eV}$ incident energy. 


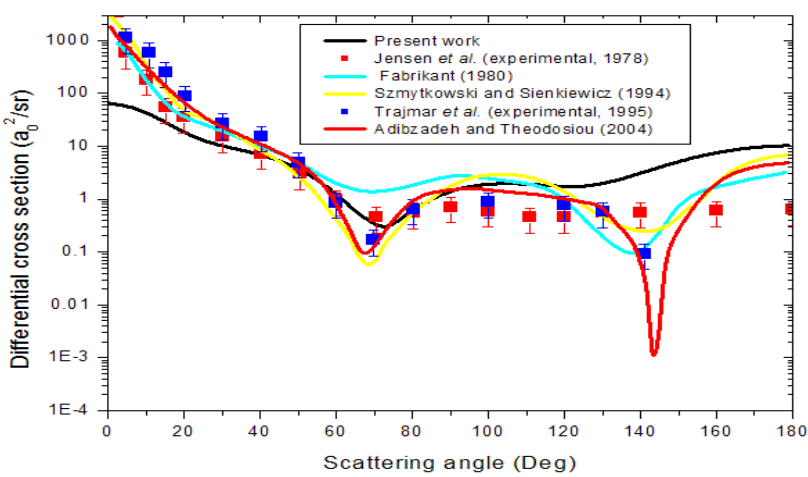

Figure 4. Differential cross section for elastic scattering of electron by barium atom at $30 \mathrm{eV}$ incident energy.

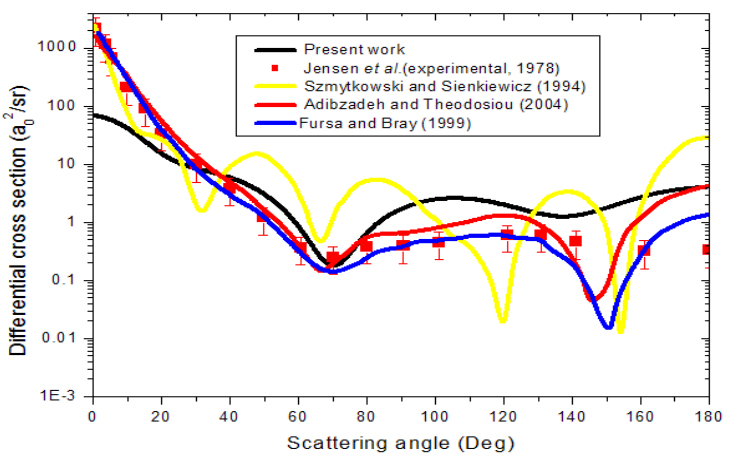

Figure 5. Differential cross section for elastic scattering of electron by barium atom at $40 \mathrm{eV}$ incident energy.

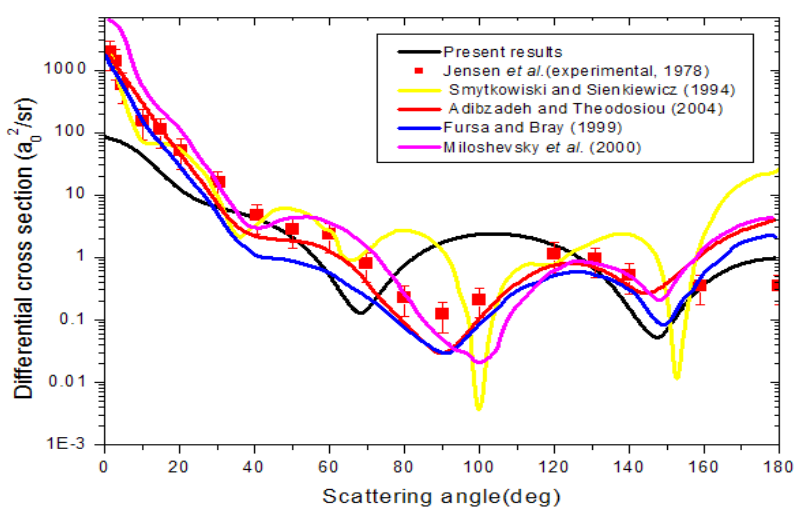

Figure 6. Differential cross section for elastic scattering of electron by barium atom at $60 \mathrm{eV}$ incident energy.

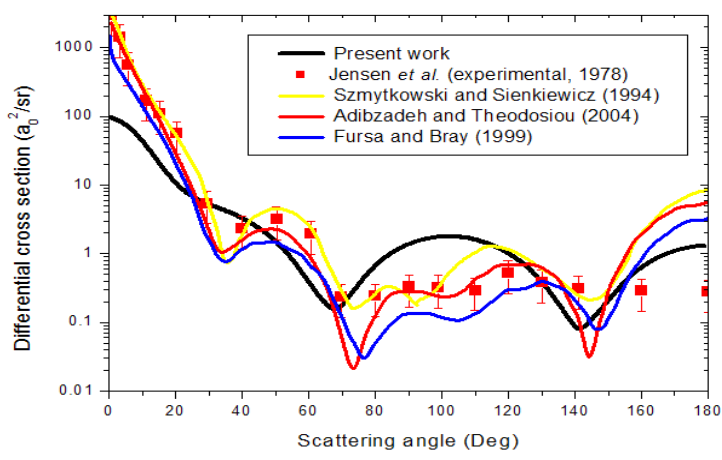

Figure 7. Differential cross section for elastic scattering of electron by barium atom at $80 \mathrm{eV}$ incident energy.
At $10 \mathrm{eV}$ (Figure 1) the present differential cross sections are in qualitative agreement (though there is quantitative difference) with results of Fursa and Bray (1999) but not with the potential scattering results of Adibzadeh and Theodosiou (2004). The present results have two minima at $50^{\circ}$ and $130^{\circ}$ as those of Fursa and Bray (1999) appearing at $60^{\circ}$ and 11 but deeper than the present ones. Adibzadeh and Theodosiou (2004) have three minima.

At $15 \mathrm{eV}$ (Figure 2) the present results are in some qualitative agreement with the experimental results of Wang et al. (1994). Both results have two minima. However, the present calculated results' first minimum is shallow and the second one has been shifted toward the left as compared with the measured results of Wang et al. (1994). This can be attributed to the approximations made in the method. Convergent close coupling results of Fursa and Bray (1999) and potential scattering results of Adibzadeh and Theodosiou (2004) also have two minima. Quantitatively the present results are higher for angles greater than $120^{\circ}$ compared to the measured results of Wang et al. (1994) as well as the calculated results of Fursa and Bray (1999) and Adibzadeh and Theodosiou (2004). This is because $15 \mathrm{eV}$ is considered to be low impact energy and DWM is not expected to give good results at low energies. The qualitative agreement indicates that the distortion potentials in this study are appropriate.

At $20 \mathrm{eV}$ (Figure 3) the present results are in better qualitative agreement up to $120^{\circ}$ with the measured results of Wang et al. (1994) than those of Jensen et al. (1978). Since Wang et al. (1994) seem to be more reliable (since it is the latest result) it shows the accuracy of the present approximation method. The present results have two minima at $70^{\circ}$ and $110^{\circ}$ compared to those of Wang et al. (1994) appearing at $30^{\circ}, 80^{\circ}$ and $140^{\circ}$. The one appearing at $30^{\circ}$ is very shallow but those at $80^{\circ}$ and $140^{\circ}$ are both deeper. The calculated results of Fursa and Bray (1999), Adibzadeh and Theodosiou (2004) and Szmytkowski and Sienkiewicz (1994) also have two minima but minima in the results of Szmytkowski and Sienkiewicz (1994) and Adibzadeh and Theodosiou (2004) are deeper compared with the present results.

At $30 \mathrm{eV}$ (Figure 4), for angles between $10^{\circ}$ to $120^{\circ}$ the present results are in satisfactory agreement with the measured results of Jensen et al. (1978) and Trajmar et al. (1999) and the CC2 results of Fabrikant (1980), the relativistic polarized orbital results of Szmytkowski and Sienkiewcz (1994) and the potential scattering results of Adibzadeh and Theodosiou (2004). The present minimum at $70^{\circ}$ almost coincides in position and depth with measured results. According to Miloshevsky et al. (2000) both the depth and positions of minima in DCS 
have significant physical importance since they reflect the structural information of the targets. This agreement signifies the accuracy of the present approximation method. For angle greater than $120^{\circ}$ the present DCS values are slightly higher than those of Jensen et al. (1978). This is because, within this range $\left(130^{\circ}-180^{\circ}\right)$, their results were obtained by probably incorrect extrapolation (Szmytkowski \& Sienkiewicz, 1994).

At $40 \mathrm{eV}$ (Figure 5) the present results are in satisfactory agreement with measured results of Jensen et al. (1978) and calculated results by Fursa and Bray (1999) and Adibzadeh and Theodosiou (2004) for angles between $20^{\circ}$ to $80^{\circ}$. The minima at $70^{\circ}$ coincides in position and depth with measured results of Jensen et al. (1978). For angles greater than $80^{\circ}$ the present DCS values are higher than those of the measured results of Jensen et al. (1978) and theoretical results of Fursa and Bray (1999) and Adibzadeh and Theodosiou (2004). The present results are in poor agreement with the calculated results of Szmytkowski and Sienkiewicz (1994), having the oscillatory behaviour which is not seen in any of the calculated and experimental results. The minimum at $140^{\circ}$ is shallow compared to other theoretical results.

At $60 \mathrm{eV}$ (Figure 6) the present results have two minima, a shallow first minimum at $70^{\circ}$ and a slightly deeper one at $150^{\circ}$. The present results are in qualitative agreement with the measured results of Jensen et al. (1978) and all the available theoretical results except that of Szmytkowski and Sienkiewicz (1994) which is too oscillatory. All the experimental and theoretical results including the present one show two minima except the one due to Szmytkowski and Sienkiewicz (1994).

At $80 \mathrm{eV}$ (Figure 7) the present results are in quantitative as well as qualitative agreement (except in the range of $80^{\circ}$ $-120^{\circ}$ ) with measured results of Jensen et al. (1978). The present results have two minima as do the measured results. The first minimum at about $70^{\circ}$ coincides in position and depth with the measured one. The present results predict a deeper second minimum at $140^{\circ}$ compared to the minimum at $110^{\circ}$ than is predicted by the measured results. The present results are in poor qualitative agreement with the calculated results of Fursa and Bray (1999), Adibzadeh and Theodosiou (2004) and Szmytkowski and Sienkiewicz (1994) since they predict more than two minima, but the differential cross section values of the present results are in the same range as other calculations.

At $100 \mathrm{eV}$ (Figure 8) it is seen that the present results above $20^{\circ}$ are in better agreement with the measured results of Jensen et al. (1978) compared to the calculated results of Symytkowski and Sienkiewicz (1994) and Miloshevsky

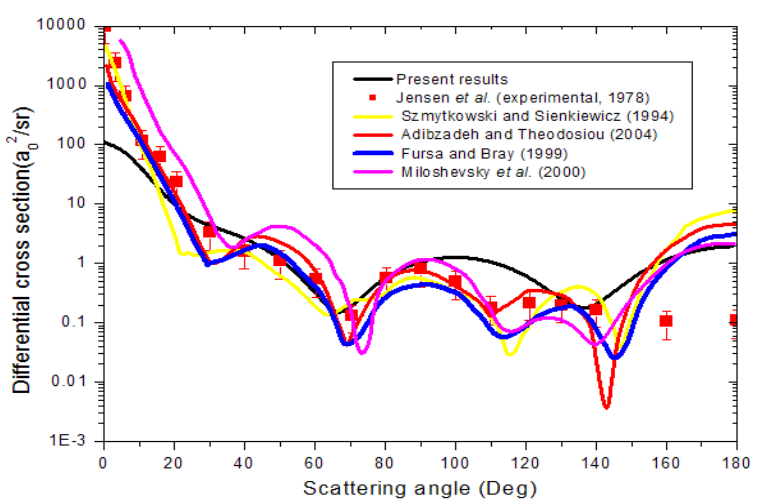

Figure 8. Differential cross section for elastic scattering of electron by barium atom at $100 \mathrm{eV}$ incident energy.

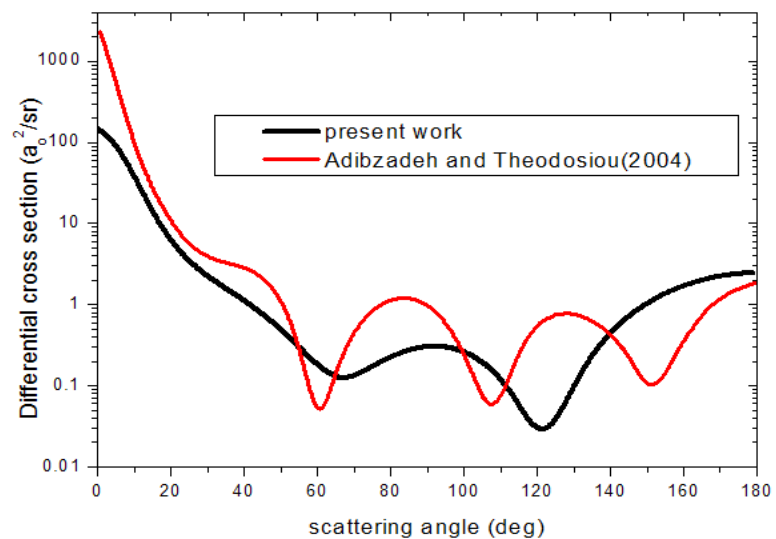

Figure 9. Differential cross section for elastic scattering of electron by barium atom at $200 \mathrm{eV}$ incident energy.

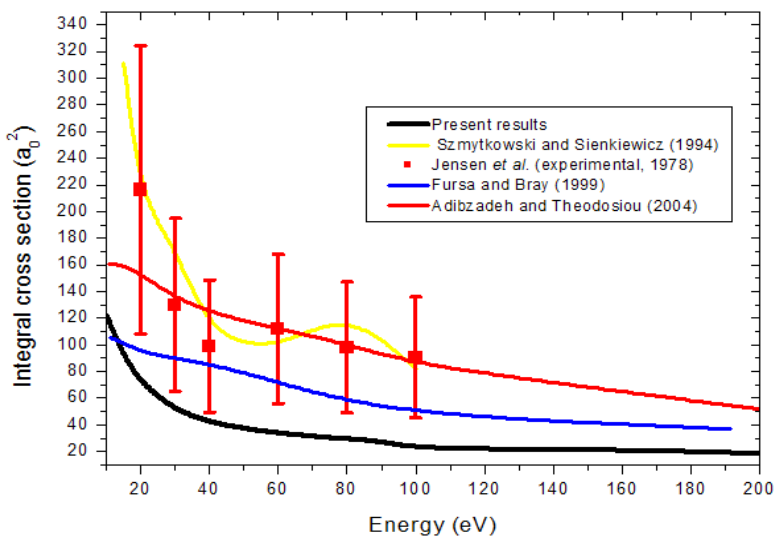

Figure 10. Integral cross section for elastic scattering of electron by barium atom.

et al. (2000). The present results have two minima as do the measured results of Jensen et al. (1978). The position and depth of the first minimum almost coincides with the measured results of Jensen et al. (1978), while the second minimum has the same depth as the experimental results, but the position is shifted. The present results are in the same range as the other theoretical results, but the present result has two minima while the other calculated results have more than two minima. It is encouraging to note that the present model is capable of reproducing the DCSs that are in good accord with measured results. 
At $200 \mathrm{eV}$ (Figure 9) the present results are compared with the only available calculated results of Adibzadeh and Theodosiou (2004). Both results fall in the same range but there is no qualitative agreement.

At all impact energies, for small scattering angles the present DCS values are lower than the available results. This is due to the exclusion of the polarization potential in this study which has a great influence on the cross sections at small scattering angles (Kariuki et al., 2015).

In the present study the integral cross section has been calculated in the range of $10-200 \mathrm{eV}$. The present results have been compared with the available theoretical and experimental data. From Figure 10 it is seen that except for the results of Szmytkowski and Sienkiewicz (1994), the present integral cross sections together with the measured results of Jensen et al. (1978) and calculated results of Fursa and Bray (1999) and Adibzadeh and Theodosiou (2004) show the same trend. That is, as energy increases the cross section decreases because the interaction time between the incident electron and target atom decreases, hence the probability of scattering decreases. Though the present results have the same trend, they are lower compared to other theoretical results.

\section{CONCLUSION}

We have compared our results with the available theoretical and experimental results. We observe that for impact energies between $30-100 \mathrm{eV}$ the present DCSs are in satisfactory agreement with the experimental results of Jensen et al. (1978) and theoretical results. This shows that the present results are reliable at intermediate and higher energies. The present integrated cross section results are also observed to be in qualitative agreement with the experimental results of Jensen et al. (1978) and the calculated results of Fursa and Bray (1999) and Adibzadeh and Theodosiou (2004). However, the present integral cross section results are lower than the available results which is due to the exclusion of a polarization potential in the distortion potential used which has a great influence on the DCS at small scattering angles.

\section{ACKNOWLEDGEMENT}

We thank the Department of Physics, Kenyatta University for providing the facility to work on this project.

\section{REFERENCES}

Adibzadeh, M., \& Theodosiou, C. E. (2004). Elastic electron scattering from $\mathrm{Ba}$ and Sr. Physical Review A, $70,052704$.

Fabrikant, I. I. (1980). Calculation of electron scattering cross Section from magnesium and barium atoms. Journal of Physics B: Atomic and Molecular Physics, 13, 603.

Fursa, D. V., \& Bray, I. (1999). Calculation of electron scattering from ground State of barium. Physical Review A, 59, 282.

Jensen, S., Register, D., \& Trajmar, S. (1978). Elastic and inelastic scattering cross section from barium. Journal of Physics B. Atomic and Molecular Physics, 11, 2367.

Kariuki, P. K., Okumu. J., \& Singh, C. S. (2015). Distorted wave Born and Optical potential calculation methods for electron-sodium elastic scattering cross sections at intermediate energies. The African Review of Physics, 10, 0018.

Kelemen, V. I., Remeta, E. Yu., \& Sabad, E. P. (1995). Scattering of electron by $\mathrm{Ca}, \mathrm{Sr}, \mathrm{Ba}$, and $\mathrm{Yb}$ atoms in the $0-200 \mathrm{eV}$ energy region in the optical model. Journal of Physics B: Atomic, Molecular and Optical Physics, 28, 1527.

Kumar, P., Jain, A. K., Tripathi, A. N., \& Nahar, S. N. (1994). Spin polarization and cross section of electron elastically scattered from heavy alkaline earth atoms. The European Physical Journal, 30, 149.

Madison, D. H., \& Bartschat, K. (1996). The distorted wave method for elastic scattering and atomic excitation. In Computational Atomic Physics. Ed. K. Bartschat. Springer, Berlin.

McLean, A. D., \& McLean, R. S. (1981). RoothanHartree-Fock atomic wave function slater basis set expansion for $\mathrm{Z}=55-92$. Atomic Data and Nuclear Data Tables, 26, 197.

Miloshevsky, G. V., Tockach, V. I., Rozan, S., \& Shanin, G. (2000). Elastic scattering of electron by gadolinium and barium atoms. Nuclear Instruments and Methods in Physics Research B, 168, 467.

Szmytkowski, R., \& Sienkiewicz J. E. (1994). Elastic scattering of electron by strontium and barium atom. Physical Review A, 50, 4007.

Trajmar, S., Kanik, I., Khakoo, I., Leclair, M. A., Bray, L., Fursa, D., \& Csanak, G. (1999). Elastic electron scattering by excited barium atom. Journal of Physics $B$ : Atomic, Molecular and Optical Physics, 32, 2801.

Wang, S., Trajmar, S., \& Zetner, P. W. (1994). Cross sections for electron scattering by ground state $\mathrm{Ba}$ : Elastic and excitation of $6 \mathrm{~s} 6 \mathrm{p}^{1} \mathrm{p}_{1}$ level. Journal of Physics B, Atomic, Molecular and Optical Physics, 27, 1613. 